\title{
Dichotomous On-Surface Self-Assembly of Tripod Molecules with Anchor Like Interaction Pattern
}

\author{
Paweł Szabelski ${ }^{1} \cdot$ Wojciech Rżysko² . Damian Nieckarz ${ }^{1}$
}

Published online: 30 April 2018

(c) The Author(s) 2018

\begin{abstract}
Recent development of methods to fabricate low dimensional molecular structures has enabled tailoring their architecture using building blocks with suitably encoded structural and chemical properties. In this contribution we study structure formation in adsorbed assemblies comprising model tripod molecules equipped with terminal arm segments providing short-range directional interactions. The interaction directions were assigned in a specific anchor like pattern which enabled the creation of diverse intermolecular connections. To explore the on-surface self-assembly of these functional units the coarse grained lattice Monte Carlo simulation method was used in which the molecules were represented as collections of interconnected segments adsorbed on a triangular lattice. Our theoretical investigations focus on the effect of symmetry and size of molecular backbone on the outcome of the 2D self-assembly. The simulations revealed the formation of complex superstructures of two types including porous networks with diverse nanocavities and nanoribbons characterized by a constant width. Occurrence of these different assemblies was found to be strongly dependent on the molecular symmetry and aspect ratio, highlighting the decisive role of the arm length distribution in the tripod building block. The theoretical results of this study can be helpful in designing new low-dimensional superstructures which are stabilized be weak intermolecular interactions as well as by covalent bonds formed in on-surface reactions such as the Ullmann coupling.
\end{abstract}

Keywords Self-assembly $\cdot$ Adsorption $\cdot$ Monte Carlo simulation $\cdot$ Pattern formation $\cdot$ Functional molecules

\section{Introduction}

Custom synthesis of organic molecules with predefined size, shape and functionality provides diverse building blocks which have been used to create low-dimensional superstructures with emergent physico-chemical properties. A versatile method in this area is the on-surface self-assembly of molecular tectons which can be carried out in both ultra vacuum conditions and at the liquid/solid interface [1,2]. A distinct advantage of this approach is the increased control over molecular connections formed between adsorbed molecules. In this case the reduced dimensionality of the

Paweł Szabelski

szabla@vega.umcs.lublin.pl

1 Department of Theoretical Chemistry, Maria-Curie Skłodowska University, Pl. M.C. Skłodowskiej 3, 20-031 Lublin, Poland

2 Department for the Modeling of Physico-Chemical Processes, Maria-Curie Skłodowska University, Pl. M.C. Skłodowskiej 3, 20-031 Lublin, Poland confining environment (adsorbing surface) facilitates correct positioning of the molecules which then can form bonds with assumed directionality and strength. The reduced rotational and translational freedom of the adsorbed molecular units is thus a factor enabling the creation of new superstructures whose synthesis in a bulk phase is normally impossible or hardly limited [3].

To date numerous examples of adsorbed structures fabricated using functional organic building blocks have been reported, in which linear [4], bent-rod [5] and star shaped [6-10] units were adsorbed on such substrates as metallic surfaces (gold, silver, copper) and highly oriented pyrolytic graphite (HOPG). Depending on the chemistry of functional groups attached to these building blocks, the resulting superstructures were sustained by interactions like, for example, hydrogen bonding $[4,6,7,9,11]$, metal-ligand coordination $[9,12]$ or van der Waals interactions $[8,10]$. Among these superstructures the nanoporous networks with regular hexagonal, square, triangular and other void spaces have been the subject of intense studies [13]. The great interest in these ordered porous architectures originates mainly 
from their potential application as versatile platforms for selective reversible/irreversible immobilization of guest species with unique optical, magnetic or catalytic properties. Such complex matrices with active centers distributed in a periodic, spatially controlled manner can serve as functional arrays with programmable response to external stimuli [14]. Moreover the nanovoids of these layered materials can provide confinement for stereoselective chemical reactions whose products can be formed only under special external geometric constraints [15].

Another type of 2D molecular structures which have been obtained from the surface confined self-assembly of organic functional building blocks are the covalently bonded systems on densely packed metallic substrates [e.g. $\mathrm{Ag}, \mathrm{Cu}$ or $\mathrm{Au}$ (111)]. A very effective approach to these superstructures has been the Ullmann coupling reaction in which appropriately halogenated tectons, usually aromatic hydrocarbons, undergo polymerization via scission of the terminal halogen atoms $[3,16,17]$. It has been demonstrated that a suitable distribution of the halogens in the monomer, either rod-like or star shaped, can result in the formation of such structures as polyphenyl chains [18], cyclic oligomers [19], ladders [20], networks [21] and graphene nanoribbons (GNRs) [22, 23]. The on-surface synthesis of the GNRs has recently gained considerable attention as the ribbons of adjustable constant width can be produced using suitably designed organic precursors. This advantage allows for tuning electronic properties of the GNRs which strongly depend of chirality, width and edge topology of these carbonaceous nanomaterials which have immense potential application in nanoelectronics.

A common task in the controlled creation of adsorbed structures sustained by weak and strong (covalent) intermolecular bonding is the choice of the optimal building block able to form superstructures with predefined morphology. This refers to such molecular properties as size, shape/aspect ratio and intramolecular distribution of interaction centers (functional groups, halogen atoms etc.). It has been often observed experimentally, that even small changes in these parameters can result in the formation of $2 \mathrm{D}$ assemblies with entirely different morphologies [24]. For that reason, identification of the molecule-superstructure relation is an important stage in custom designing and fabrication of molecular superstructures on solid substrates. In practice, information of this kind is usually obtained in a series of test experiments in which different molecular candidates are probed. As this screening procedure requires synthesis of numerous molecular tectons and subsequent imaging of the resulting assemblies (usually with scanning probe microscopy), the selection of the optimal building block can be time and resource consuming.

An alternative approach which enables facile optimization of molecular systems on surfaces are the computer simulations $[9,11,24-31]$. This technique is particularly useful, as it makes it possible to study readily the effect of intrinsic molecular properties on the corresponding superstructures formed under variable conditions (e.g. temperature, density). When a simplified coarse grained representation of the modeled system is used, like for example in the lattice Monte Carlo (MC) simulations [9, 11, 25-27], main structural and thermodynamic properties of adsorbed assemblies can be studied in a relatively short time. An additional advantage of the lattice MC calculations is the generality, by which a wide class of chemically different molecules having the same shape (e.g. tripod, cross etc.) and distribution of interaction centers can be modeled using one common unit structure. This strategy was successfully applied to numerous functional molecules including aromatic tricarboxylic acids [25-27], tripods equipped with terminal pyridyl groups $[10,12]$, derivatized dehydroxybenzoannulenes (DBAs) [32], ditopic rodlike molecules with end cyano groups [33] and others [20, 34]. In the aforementioned examples very good matches between the simulated results and their experimental counterparts were obtained highlighting validity of the coarsegrained self-assembly model. Moreover, the simulations revealed to be able to predict new complex molecular patterns, such as, for example, the metal-organic Sierpiński triangle fractal [34], whose existence was later confirmed experimentally [35].

In our previous studies the MC simulation method was used to decipher the role of directional short-range interactions on the surface-assisted self-assembly of tripod molecules on a solid substrate having (111) symmetry [36-38]. An interesting effect was observed for the molecules with reduced backbone symmetry and interaction directions forming anchor like pattern. For those molecules the creation of complex porous networks and ribbons was observed, depending on the molecular aspect ratio [39]. In this contribution we present a systematic study on the influence of molecular geometry on the outcome of the 2D self-assembly of these tectons. To that end we examine how changing of the length of molecular arms of the tripod building block affects structure formation in the adsorbed overlayer. The main objective of our theoretical investigations is to identify key factors which would allow for directing the self-assembly towards porous networks and nanoribbons. The results of the MC modeling can be helpful in preliminary screening of molecular libraries to select functional units able to create superstructures having the two distinct morphologies. Moreover, if covalently bonded nanostructures are considered, our model can describe the formation of the corresponding precursor metal-organic structures of the Ullmann coupling reaction. 


\section{The Model and Simulation}

The tripod molecules of our model were treated as flat rigid structures comprising a few interconnected segments, each of which was allowed to occupy one site on a triangular lattice, as shown in Fig. 1. These building blocks consisted of a central segment called core and three attached arms of adjustable length, measuring $a, b$ and $c$ segments, respectively. Only the terminal arm segments of the tripod were active, that is able to interact with neighboring foreign active segments. The active segments were assigned unique interaction directions forming anchor like pattern shown in the figure (red arrows). Specifically, the interaction directions assigned to the arms $a$ and $c$ were rotated by $\pm 60^{\circ}$ with respect to the arms, respectively. In the case of the arm $b$ the interaction direction was collinear with the arm. This assignment can correspond to the distribution of functional groups in the exemplary organic molecule sketched in the right part of Fig. 1. Here, the assumed anchor pattern can be realized using functional groups in positions meta ( $a$, $c)$ and para (b) of the terminal phenyl ring. The interactions between adsorbed molecules were characterized by a short range segment-segment interaction potential limited to nearest neighbors on a triangular lattice. The interaction was possible only for neighboring active segments whose interaction directions were collinear $(\rightarrow \leftarrow)$. In this case the interaction energy, $\varepsilon$ was equal to -1 . For the remaining molecular configurations this energy was assumed to be equal to zero. The adsorbate density, $\rho$ was defined as the average number of molecular segments per one lattice site, that is $\rho=N(a+b+c+1) / L^{2}$ where $N$ is the number of

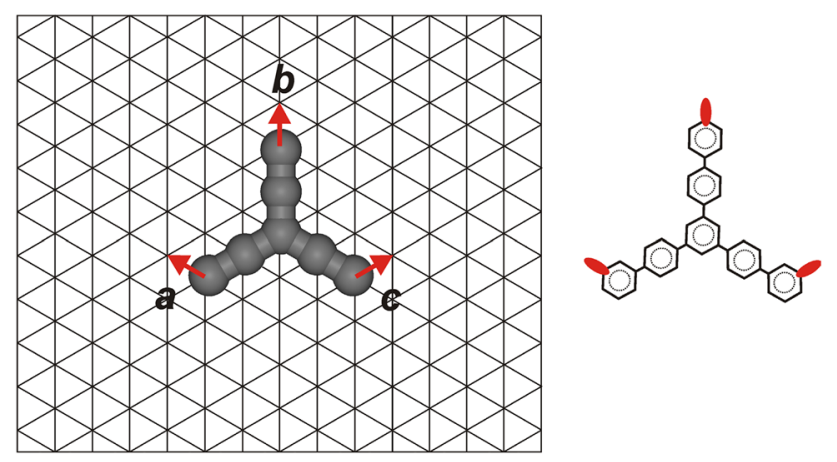

Fig. 1 (left) Schematic structure of the model tripod-shaped molecule with anchor like interaction pattern, adsorbed on a triangular lattice. The red arrows indicate the corresponding directional segment-segment interactions assumed in the model. The molecule shown in the figure consists of three arms of adjustable length: $a, b$ and $c$ segments. In the case considered here $a=b=c=2$. (right) An example of the corresponding tripod organic molecule with functional groups (red, meta, para, meta) distributed in a way providing the directional interactions adsorbed molecules. The energies and temperatures in our model are reduced quantities expressed in units of $\varepsilon$ and $|\varepsilon| / k$, respectively with $k$ being the Boltzmann constant.

The simulations were performed on a rhombic fragment of a triangular lattice of equivalent adsorption sites with side, $L$ equal to 384 and 672 sites. According to the assumed energetic homogeneity of the adsorbing surface, for convenience, the surface-molecule interaction energy was set equal to zero. To minimize edge effects periodic boundary conditions were used in both planar directions. The calculations were carried out using the lattice Monte Carlo simulation method with the conventional Metropolis acceptance scheme. To avoid trapping of the modeled systems in metastable states the parallel tempering (PT) technique was applied, [37, 40] in which four system replicas (having the same $\rho$ ) were simulated at four evenly spaced temperatures ranging from 0.12 to 0.18 or from 0.1 to 0.25 . To equilibrate the adsorbed assemblies we used $10^{8} \mathrm{MC}$ steps each of which was defined as a single attempt to move a randomly selected molecule to a new position. Translation of the molecule was accompanied by random in-plane rotation around its core, by multiple of 60 degrees. In the PT procedure the configurations corresponding to two randomly selected temperatures from a given set were swapped every $5 \times L^{2}$ MC steps. To calculate the results presented in the following sections further $10^{9} \mathrm{MC}$ steps were performed.

\section{Results and Discussion}

\subsection{Molecules with $C_{3}$-Symmetric Backbone}

To assess the effect of backbone shape on the self-assembly of the tecton from Fig. 1 we examined a series of test cases differing by $a, b$ and $c$. In the simplest situation $C_{3}$-symmetric molecules were used with $a=b=c=1,2$ and 3 . These building blocks are further called 111, 222 and 333, according to the $a b c$ code adopted here. Figure 2 presents representative snapshots of the adsorbed overlayers in which the formation of ordered extended molecular patterns was observed. At sufficiently low adsorbate density ( 0.25 for 111 and 0.1 for 222 and 333, top panels) the molecules create complex openwork superstructures in which three types of nanocavities can be distinguished, including large rounded pores and smaller elongated and hexagonal ones. The networks obtained for the three tectons are very similar in terms of spatial organization being isostructural and they contain pores with size that scales with the arm length of the building block, $n=a=b=c$. For example, the area of the largest pore, $A$ defined as the number of empty lattice sites within the pore, can be expressed as $39 n^{2}+18 n+1$. Regarding the periodicity of the simulated structures, it should be pointed out that they are often locally periodic comprising 


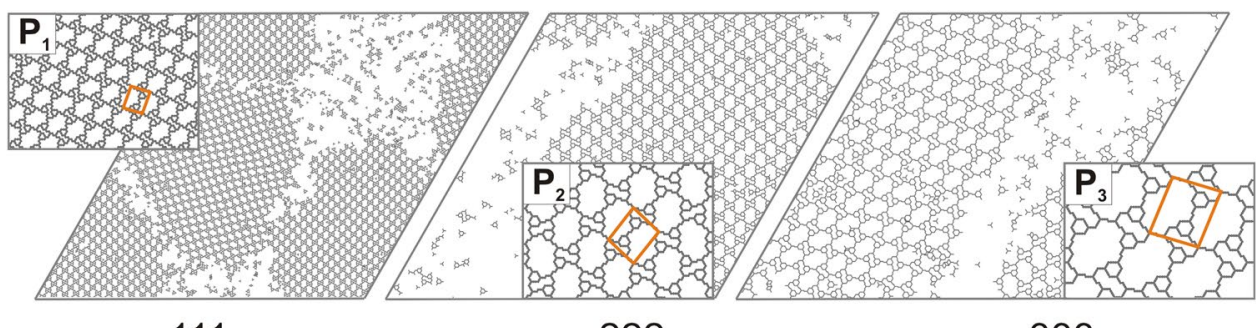

111

222

333



Fig. 2 Snapshots of the adsorbed overlayers $(L=384)$ comprising the molecules 111, 222 and 333. The structures shown in the top row correspond to the lower adsorbate density $(0.25, T=0.18 ; 0.10$, $T=0.16$ and $0.10, T=0.16$, respectively) while these from the bottom row were obtained at the increased value of $\rho(0.40, T=0.18$;

interconnected domains or rows of (large) pores which are rotated with respect to each other but nevertheless create extended continuous networks. In the insets in the top part of Fig. 1. we presented ordered phases, $P_{n}$ which can be found in the obtained overleyers, along with the corresponding unit cells marked in orange. When the adsorbate density is increased ( 0.40 for $111,0.2$ for 222 and 0.15 for 333, bottom panels), the new denser chevron-like pattern $D_{n}(n=1 \ldots 3)$ emerges, regardless of the tecton size and it is characterized by much lower structural complexity compared to $P_{n}$. In this case, the simulated networks comprise pores of one type, resembling elongated parallelograms whose area, $A$ (number of empty sites) scales with $n$ as $9 n^{2}-1$. Note that pores of the above type are also present in the corresponding networks $P_{n}$ in which they play the role of (four) corner elements of the largest pores. In this context the structural transformation $P_{n} \rightarrow D_{n}$ which occurs due to the increase in the adsorbate density involves elimination of the largest and smallest pores of $P_{n}$, so that the medium-sized pores with elongated shape are exclusively preserved in the resulting network $D_{n}$. Such an effect means increase of the homogeneity of the network in terms of pore size/shape distribution. Moreover the $D_{n}$ patterns are globally periodic.

To quantify the effect of the arm length on the structural properties of the simulated networks, in Fig. 3 we plotted the dependence of the area of the largest pore of $P_{n}$ and $D_{n}$ on the parameter $n$, obtained using the simple functional relations discussed earlier. Moreover, to compare further individual properties of the obtained networks, in the figure we showed also how the network density, $d$ defined as the number of occupied lattice sites per unit cell area of $P_{n}$ and
$0.2, T=0.16$ and $0.15, T=0.18$, respectively). The insets are the magnified fragments of the ordered phases $P_{n}$ and $D_{n}(n=1 \ldots 3)$ with the corresponding unit cells marked in orange. The red and blue lines indicate alternating rows of elongated pores (shown for $D_{1}$ ) occurring in the chevron patterns $D_{n}$

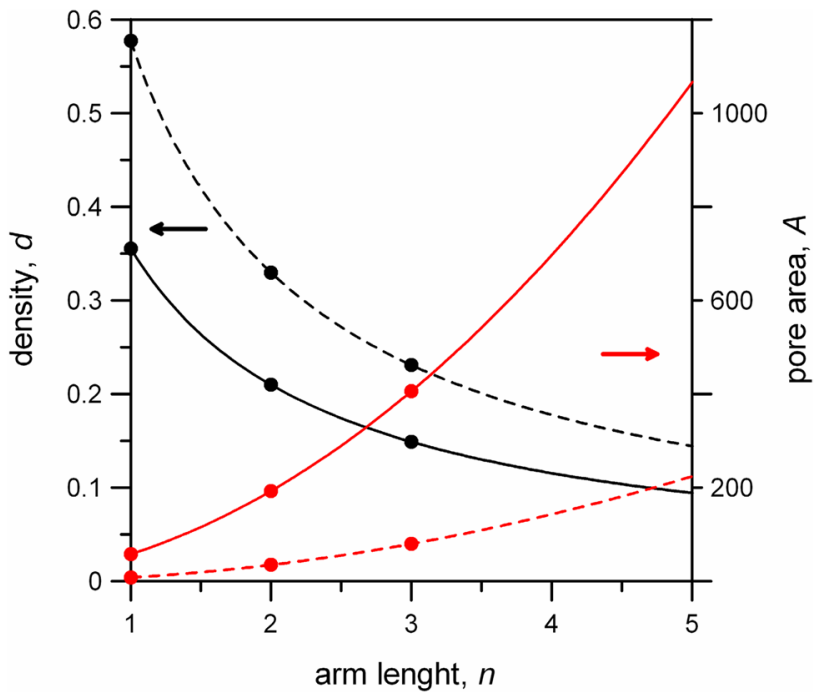

Fig. 3 Effect of the arm length of the tripod building block on the density, $d$ and area of the largest pore, $A$ of the corresponding networks $P_{n}$ (solid lines) and $D_{n}$ (dashed lines). The points correspond to the networks shown in Fig. 2

$D_{n}$, changes with the arm length of the tripod building block. The corresponding functional dependencies for $d(n)$ are given by: $8 /[(9 n+4) \sqrt{3}]$ and $4 /[(3 n+1) \sqrt{3}]$, respectively.

As it follows from Fig. 3, the area of the largest pore in $P_{n}$ increases much stronger with increasing arm length, as compared to $D_{n}$. The main reason for this effect is that the (largest) pores occurring in the network of the first type are surrounded by 12 molecules each while the elongated pores forming $D_{n}$ engage only 6 molecules. In consequence, an 
increase in the arm length, for example by one segment, leads to the corresponding increase of the pore perimeter but this effect is more strongly amplified for the pores with 12-membered rims. Accordingly, for these largest nanocavities the associated pore area grows much faster with $n$ compared to the elongated pores with 6-membered rims. For example, the pore area calculated for $P_{3}$ is about five times larger than that obtained for $D_{3}(n=3$ in Fig. 3). On the other hand, changing of the arm length does not lead to such profound differences between the overall network densities, $d(n)$ calculated for the corresponding superstructures. In this case, the largest difference (distance between the corresponding curves) can be observed for the smallest tecton $(n=1)$ and this quantity decreases slowly with increasing $n$. As in the $P_{n}$ networks, the largest pores are accompanied by the smallest hexagonal pores with denser molecular packing, the overall density of $P_{n}$ is not dramatically higher than that of $D_{n}$ which does not contain the smallest pores. This effect is especially visible at the higher values of $n$.

\subsection{Molecules with Lower Backbone Symmetry}

To assess the effect of the reduced backbone symmetry on the structure formation in the modeled systems we next performed the simulations for the tectons $a b c$ in which the middle arm $(b)$ was longer than the two remaining ones having the same length $(a=c)$. The main objective in this case was to test whether the dichotomy observed previously for the self-assembly of the tectons 121 and 212 is a general rule which applies also to analogous pairs of building blocks [39]. To that end the calculations were carried out at constant adsorbate density $\rho=0.15$, for the molecules 131 , 232 and additionally, for comparative purposes, for the unit 121. Figure 4 presents the corresponding snapshots obtained for these three building blocks.

The main conclusion from the results shown in Fig. 4 is that all of the considered molecules $(121,131,232)$ create extended porous networks and that these networks are isostructural. Moreover, the networks from the figure are also isostructural with the $P_{n}$ s obtained previously for the $C_{3}$ -symmetric tectons 111, 222 and 333 (compare with Fig. 2). Specifically, all of the networks of $P_{n}$-type discussed so far consist of pores of three types, regardless of the assumed molecular aspect ratio. These are the largest pores formed by 12 molecules, medium-sized elongated pores with 6-membered rims and the smallest pores formed by 3 molecules. The spatial distribution of these pores in the $P_{n}$ networks is identical, that is each largest pore have four neighbors of the same kind and its walls are formed by pairs of smaller pores of the same shape. An easily noticeable effect here is the change in the shape proportions of the contributing pores which is induced by elongation/shortening of the molecular arms. For example, the shape of the smallest pores becomes more triangular as the ratio $b /(a=c)$ increases. This change produces also the largest pores with more elongated shape, as it can be seen for the tecton 131. The obtained networks, like those corresponding to 111,222 and 333 are, in general, not fully periodic but contain the $P_{n}$ domains which are characterized by the parallelogram unit cells shown in the insets in Fig. 4.

Another type of lowered asymmetry that can be introduced to the molecule $a b c$ is the elongation of the outer arms, so that $a=c>b$. In this case, in the simulations we used the tectons 313,323 and 212 , with the last one recalled for comparative purposes [39]. Like for the asymmetric molecules discussed previously, the calculations were performed assuming the same surface coverage, that is $\rho=0.15$. Figure 5 presents snapshots of the resulting self-assembled structures. The most apparent effect which can be observed when changing the asymmetry type of the tripod tecton is the formation of molecular structures which are entirely different than the $P_{n}$ s from Fig. 4. Elongation of the two outer arms of the tripod produces ribbons comprising isostructural elementary units. These units, called $\mathrm{R}$ and $\mathrm{S}$, are mirror images of each other and always comprise eight molecules which create one larger pore with elongated shape (formed by six molecules) and two smaller pores surrounded by three molecules. The area of the largest pores of the ribbons formed by 212, 313 and 323 equals to 24, 48 and 63 lattice sites, respectively. The chiral units in the ribbons can be connected, through the interactions marked by the red arrows in Fig. 5, to form diverse sequences of the $\mathrm{R}$ and $\mathrm{S}$ units which we exemplified in the figure. In consequence, the ribbons have usually irregular edges and deviate from being perfectly linear. However, despite the various structural motifs
Fig. 4 Snapshots of the adsorbed overlayers $(L=384)$ comprising the molecules 121 , 131 and 232. The structures shown in the figure were obtained at $\rho=0.15$ and $T=0.18(121,131)$ and $T=0.16$ (232). The corresponding unit cells are marked with the orange lines
121 131 232




Fig. 5 Snapshots of the adsorbed overlayers $(L=672)$ comprising the molecules 212,313 and $323 ; \rho=0.15$, $T=0.15$ (top). The structures shown in the bottom part are the magnified fragments of the ribbons from the corresponding panels. The letters $\mathrm{R}$ and $\mathrm{S}$ denote mirror-image structural units of the ribbons connected by the directional interactions indicated by the red arrows
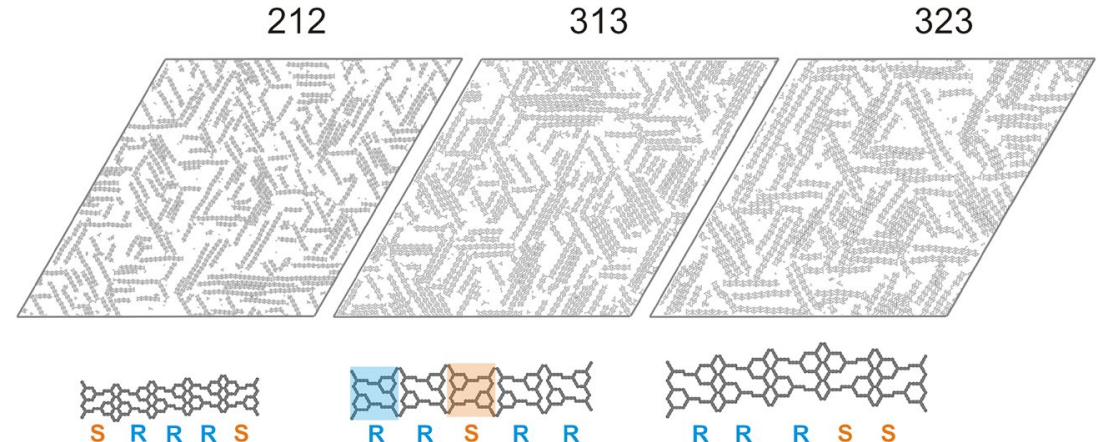



$\begin{array}{lllll}R & R & R & S & S\end{array}$ resulting from the mixed R-R, S-S and R-S connections, the ribbons preserve constant width.

The results obtained with the tectons of type $a=c>b$ and $a=c<b$ confirm that the difference in the arm length is the key structural parameter which dictates the selfassembly. As this conclusion refers to molecules with symmetrical distribution of the interaction directions it is worth examining whether it can be further generalized to include molecules in which the interaction directions are assigned differently to the arms $a, b$ and $c$. Based on the findings obtained so far, it can be expected that similar effects should be observed for the molecules for which the outer arms $a$ and $c$ do not necessary have the same length.

\section{3 (pro)Chiral Molecules}

A stringent test for the hypothesis put forward in the preceding section is to examine whether tripods with more complex structural properties are able to form the two types of self-assemblies. One class of such molecules are, for example, the units 112, 113 and 223. These molecular building blocks have the backbone shape identical with 121, 131 and 232, respectively, but according to our notation $a b c$ it is now the $\operatorname{arm} c$ which is longer by one segment than the two remaining arms having the same length $(a=b)$. This assumption lowers the symmetry of the interaction directions and makes the molecules 112,113 and 223 prochiral in terms of the possibility of bond-formation (see the insets in Fig. 6). Namely, such tectons can be adsorbed in one of two
Fig. 6 Snapshots of the adsorbed overlayers comprising the molecules ( $R$-enatiomers) 112,113 and 223 (top) and 221, 331 and 332 (bottom). The insets show magnified fragments of the corresponding molecular structures. Elongated pores of two types, occurring in each structure are shown in orange and blue. $L=384$, $\rho=0.15$ and $T=0.12$
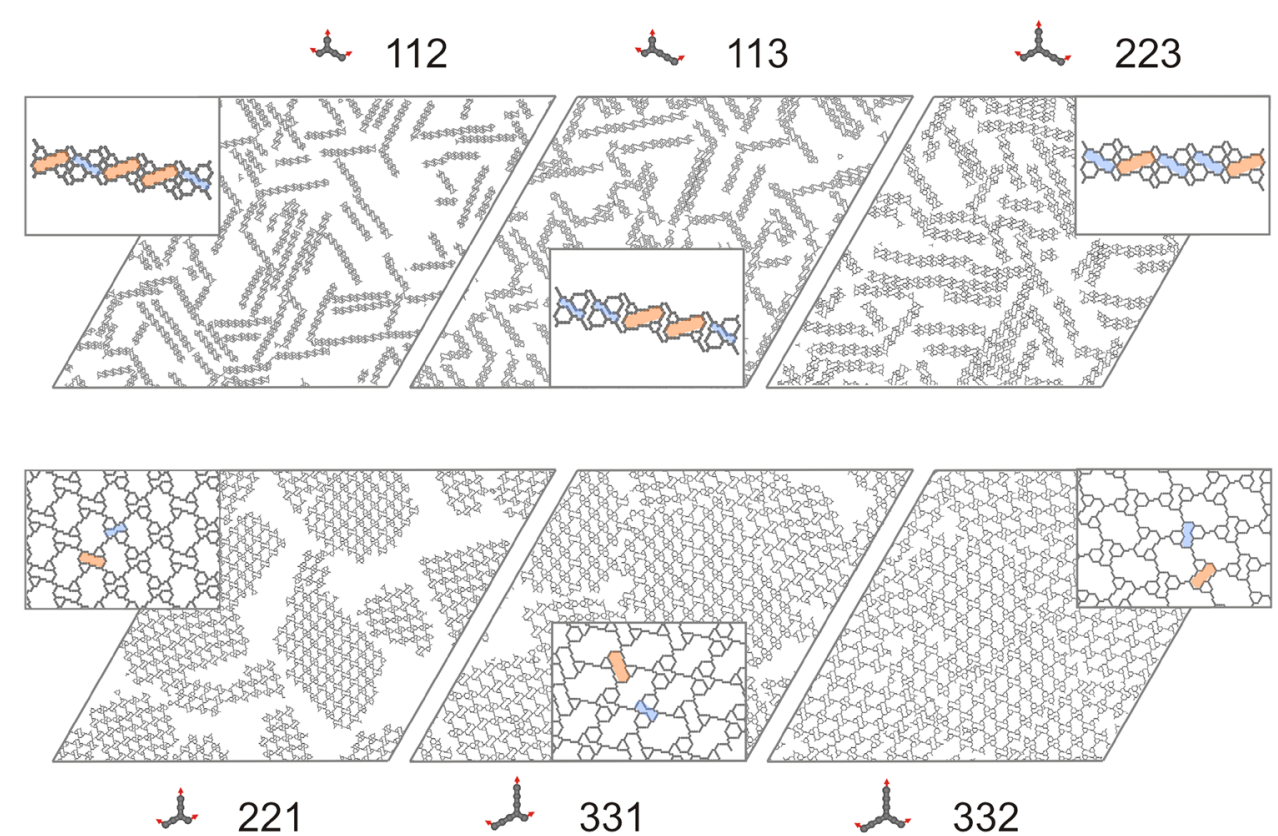
mirror-image planar configurations (surface enantiomers) of which the one called arbitrarily $R$ is shown for each building block in Fig. 6. The same situation refers to the molecules with the opposite relation between the arm lengths, that is 221,331 , and 332, for which the $\operatorname{arm} c$ is now shorter by one segment compared to two the two remaining ones $(a=b)$. The above discussion on the prochiral units can be summarized with a question on which type of the superstructures (networks vs. ribbons) can be obtained when the tripod tecton has one longer/shorter arm and this arm is not the central one, $b$. Figure 6 , in which we plotted self-assembled structures comprising the corresponding enantiomers $R$, brings answer to this question.

As it can be seen in the figure, the molecules for which $c>a, b$ create ribbons while those for which the opposite relation $c<a, b$ holds, self-assembly into extended porous networks with complex architectures. Due to the increased asymmetry of the interaction directions and molecular backbone, the structures presented in Fig. 6 are in general more structurally heterogeneous compared to their counterparts obtained previously (compare with Figs. 4, 5). The ribbons formed by the molecules of the first group (112, 113 and 223) contain elongated pores which are different in size (marked in blue and orange). The structural units, containing these pores, and similar to those shown in Fig. 5, are often connected in a random sequence so that the elongated pores are distributed non-periodically along the ribbon, as shown in the insets. An interesting effect here is that, the elongated pores of one size are oriented in the same direction, and moreover the smaller (blue) and bigger pores (orange) are rotated with respect to each other by a constant angle. The area of these smaller and bigger pores in the ribbons, expressed in lattice sites, is equal to 9 and 18 for 112, 10 and 38 for 113 and 38 and 58 for 223.

For the networks presented in the bottom part of Fig. 6, the corresponding structural characteristics are even more complex, as these networks are now built of pores of six types. These are the largest pores of two irregular shapes which can be seen in the insets (see for example 331), medium-sized elongated pores of two types marked in blue and orange and the smallest hexagonal and triangular pores. Because of the diverse ways in which the pores can be connected to build the network, the resulting structures are in general aperiodic but preserve continuity. This effect can be easily noticed when looking at the position of the mediumsized elongated pores (orange and blue) in the magnified fragments shown in Fig. 6. For comparison with the elongated pores occurring in the ribbons we calculated the area of the analogous pores of the simulated networks. These values are the following: 16 and 32 for 221, 26 and 68 for 331 and 51 and 76 being in general larger than those obtained for the ribbons.

Our final approach to determine the rule governing the dichotomous self-assembly was to perform simulations for the chiral tripods having all arms of different lengths, that is for $a \neq b \neq c$. To that end we used three tectons: 132, 123 and 213 in which the length of the central arm was gradually decreased by one segment (from 3 to 1). Figure 7 presents snapshots simulated for these building blocks. As it can be seen in the figure, the molecule 132 for which the central $\operatorname{arm}(b)$ is longer than any of the two remaining arms ( $a$ and $c$ ) creates a complex porous network. Like for the chiral molecules discussed previously (see Fig. 6), the obtained network comprises pores of six types and it is not globally periodic. On the other hand, the self-assembly of the two other molecules for which the central arm is shorter than one (123) and two (213) of the remaining arms produces ribbons. The internal structure of these ribbons is similar to the structure observed for the molecules with $a=b$. The ribbons from Fig. 7 comprise elongated pores of two sizes (colored in blue in orange) which can occur in random sequences of which examples are shown in the insets. In this case, it can be observed again that the pores of one size are oriented in the same direction. The area of the pores occurring in the ribbons equals to: 17 and 53 for 123 and 27 and 43 for 213 . For comparison, analogous pores in the network formed by 132 measure 26 and 45 lattice sites respectively.

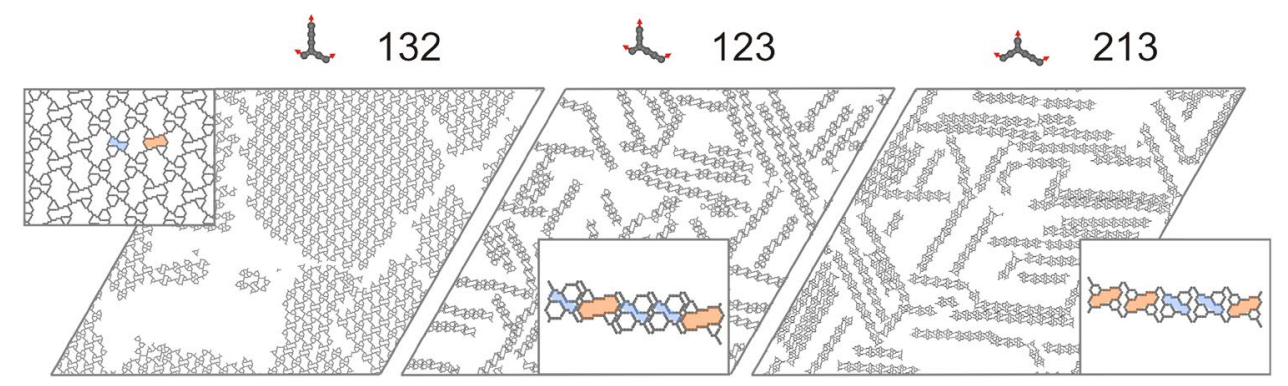

Fig. 7 Snapshots of the adsorbed overlayers comprising the molecules ( $R$-enantiomers) 132, 123 and 213 The insets show magnified fragments of the corresponding molecular structures. Elongated pores of two types, occurring in each structure are shown in orange and blue. $L=384, \rho=0.15$ and $T=0.12$ 
The result of the simulations performed for the variety of tripod shaped molecules with anchor-like interaction pattern allow us to formulate a general self-assembly principle which applies to all of the modeled systems. It can be summarized as follows: for the molecules having the central arm (b) that is not shorter than any of the remaining arms the outcome of the self-assembly are the porous networks. In the opposite case, that is when the central arm is shorter than at least one of the remaining arms the resulting structures are the nanoribbons. This simple structure-property relation provides hints on how to select molecular building blocks which would be able to create superstructures of the two types discussed in this work. Moreover, the obtained findings demonstrate how the porosity of the networks and ribbons (including pore shape and size) can be controlled by suitable manipulation of the relation $a: b: c$.

\section{Conclusions}

The results of this study show that molecular aspect ratio is an important factor which can strongly affect the selfassembly of functional building blocks on solid substrates. The example of a tripod molecule with the special anchorlike assignment of short range interaction directions demonstrates the possibility of directing the self-assembly towards superstructures with entirely different morphologies, including porous networks and nanoribbons. This possibility can be realized by a suitable elongation/shortening of molecular arms of the tripod building block, for example, via synthesis of organic tripods with arms comprising different numbers of interconnected phenyl rings. Our theoretical calculations show that there is a simple rule which governs the dichotomous self-assembly producing porous networks or ribbons. Specifically, a prerequisite for the molecule to create porous networks is that its central arm ( $b$, see Fig. 1) can not be shorter than any of the two remaining arms $(a, c)$. In the opposite case ribbons with diverse porous architectures are formed. The simulations performed for a wide range of molecular structures, including symmetric and asymmetric (prochiral) building blocks confirm this principle and they indicate the large pool of molecular tectons which can be potentially used to create the corresponding superstructures. Moreover, fine tuning of the aspect ratio of the model tripod molecules allows for controlling of size of the pores occurring in the resulting $2 \mathrm{D}$ assemblies. For example, this advantage can be used for designing low dimensional molecular structures resembling graphene nanonoribons with predefined porosity. In this case, the tripod tectons studied here can be treated as polyphenyl molecules with halogen atoms attached according to the anchor pattern from Fig. 1. If these molecules are further used to create metal-organic structures sustained by twofold nodes, like it normally occurs in the $\mathrm{Cu}$-assisted Ullmann coupling, the outcome of the self-assembly can be appropriately directed by tuning the molecular aspect ratio.

Acknowledgements This work was supported by the National Science Centre Poland research grant 2015/17/B/ST4/03616.

Open Access This article is distributed under the terms of the Creative Commons Attribution 4.0 International License (http://creativeco mmons.org/licenses/by/4.0/), which permits unrestricted use, distribution, and reproduction in any medium, provided you give appropriate credit to the original author(s) and the source, provide a link to the Creative Commons license, and indicate if changes were made.

\section{References}

1. Barth JV (2007) Molecular architectonic on metal surfaces. Annu Rev Phys Chem 58:375-407

2. Ciesielski A, Palma CA, Bonini M, Samorì P (2010) Towards supramolecular engineering of functional nanomaterials: preprogramming multi-component 2Dself-assembly at solid-liquid interfaces. Adv Mater 22:3506-3520

3. Lindner R, Kühnle A (2015) On-surface reactions. ChemPhysChem 16:1582-1592

4. Kühne D, Klappenberger F, Decker R et al (2009) Self-assembly of nanoporous chiral networks with varying symmetry from sexiphenyl-dicarbonitrile on $\mathrm{Ag}(111)$. J Phys Chem C 113:17851-17859

5. Shang J, Wang Y, Chen $M$ et al (2015) Assembling molecular Sierpiński triangle fractals. Nat Chem 7:389-393

6. Silly F (2014) Two-dimensional self-assembly of 2,4,6-tris (4',4",4"'-trimethyl-phenyl)-1,3,5-triazine star-shaped molecules: nanoarchitecture structure and domain boundaries. J Phys Chem C 118:11975-11979

7. Barnard RA, Dutta A, Schnobrich JK et al (2015) Two-dimensional crystals from reduced symmetry analogues of trimesic acid. Chem Eur J 21:5954-5961

8. Tahara K, Furukawa S, Ujii H et al (2006) Two-dimensional porous molecular networks of dehydrobenzo[12]annulene derivatives via alkyl chain interdigitation. J Am Chem Soc 128:16613-16625

9. Vijayaraghavan S, Écija D, Auwärter W et al (2013) Supramolecular assembly of interfacial nanoporous networks with simultaneous expression of metal-organic and organic-bonding motifs. Chem Eur J 19:14143-14150

10. Miyake K, Hori Y, Ikeda T et al (2008) Alkyl chain length dependence of the self-organized structure of alkyl-substituted phthalocyanines. Langmuir 24:4708-4714

11. Ciesielski A, Szabelski P, Rżysko W et al (2013) Concentrationdependent supramolecular engineering of hydrogen-bonded nanostructures at surfaces: predicting self-assembly in 2D. J Am Chem Soc 135:6942-6950

12. Jun L, Tao L, Ziliang S et al (2011) Structural transformation of two-dimensional metal-organic coordination networks driven by intrinsic in-plane compression. J Am Chem Soc 133:18760-18766

13. Kudernac T, Lei S, Elemans JAAW., De Feyter S (2009) Twodimensional supramolecular self-assembly: nanoporous networks on surfaces. Chem Soc Rev 38:402-421 
14. Wieghold S, Li J, Simon P et al (2016) Photoresponse of supramolecular self-assembled networks on graphene-diamond interfaces. Nat Commun 7:10700

15. Müller K, Enache M, Stöhr M (2016) Confinement properties of $2 \mathrm{D}$ porous molecular networks on metal surfaces. J Phys 28:153003

16. Lackinger M (2017) Surface-assisted Ullmann coupling. Chem Commun 53:7872-7885

17. Shen Q, Gao HY, Fuchs H (2017) Frontiers of on-surface synthesis: from principles to applications. Nano Today 13:77-96

18. Lipton-Duffin JA, Ivasenko O, Perepichka DF, Rosei F (2009) Synthesis of polyphenylene molecular wires by surface-confined polymerization. Small 5:592-597

19. Chen M, Shang J, Wang Y et al (2017) On-surface synthesis and characterization of honeycombene oligophenylene macrocycles. ACS Nano 11:134-143

20. Lin T, Shang XS, Adisoejoso J et al (2013) Steering on-surface polymerization with metal-directed template. J Am Chem Soc 135:3576-3582

21. Eichhorn J, Nieckarz D, Ochs O et al (2014) On-surface Ullmann coupling: the influence of kinetic reaction parameters on the morphology and quality of covalent networks. ACS Nano 8:7880-7889

22. Segawa $\mathrm{Y}$, Ito H, Itami K (2015) Structurally uniform and atomically precise carbon. Nat Rev Mater 1:15002

23. Cai J, Ruffieux P, Jaafar R et al (2010) Atomically precise bottomup fabrication of graphene nanoribbons. Nature 466:470-473

24. El Garah M, Dianat A, Cadeddu A et al (2016) Atomically precise prediction of 2D self-assembly of weakly bonded nanostructures: STM insight into concentration-dependent architectures. Small 12:343-350

25. Misiūnas T, Tornau EE (2012) Ordered assemblies of triangularshaped molecules with strongly interacting vertices: phase diagrams for honeycomb and zigzag structures on triangular lattice. J Phys Chem B 116:2472-2482

26. Simenas M, Ibenskas A, Tornau EE (2013) Ordering of triangular molecules into the honeycomb phase: estimation of critical temperature for different lattice models of self-assembly. Phase Transit 86:866-877

27. Ibenskas A, Tornau EE (2012) Statistical model for self-assembly of trimesic acid molecules into homologous series of flower phases. Phys Rev E 86:51118-51130
28. Copie G, Cleri F, Makoudi Y et al (2015) Surface-induced optimal packing of two-dimensional molecular networks. Phys Rev Lett 114:66101-66106

29. Palma CA, Samorì $P$, Cecchini M (2010) Atomistic simulations of 2D bicomponent self-assembly: from molecular recognition to self-healing. J Am Chem Soc 132:17880-17885

30. Van der Lit J, Marsman JL, Koster RS et al (2016) Modeling the self-assembly of organic molecules in 2D molecular layers with different structures. J Phys Chem C 120:318-323

31. Zhao Y, Wu Q, Chen Q, Wang J (2015) Molecular self-assembly on two-dimensional atomic crystals: insights from molecular dynamics simulations. J Phys Chem Lett 6:4518-4524

32. Adisoejoso J, Tahara K, Lei S et al (2012) One building block, two different nanoporous self-assembled monolayers: a combined STM and Monte Carlo study. ACS Nano 6:897-903

33. Nieckarz D, Szabelski P (2013) Understanding pattern formation in 2D metal-organic coordination systems on solid surfaces. J Phys Chem C 117:11229-11241

34. Nieckarz D, Szabelski P (2014) Simulation of the self-assembly of simple molecular bricks into Sierpiński triangles. Chem Commun 52:6843-6845

35. Sun Q, Cai L, Ma H et al (2015) On-surface construction of a metal-organic Sierpiński triangle. Chem Commun 51:14164-14166

36. Szabelski P, Rżysko W, Nieckarz D (2016) Directing the selfassembly of tripod molecules on solid surfaces: a Monte Carlo simulation approach. J Phys Chem C 120:13139-13147

37. Rżysko W, Nieckarz D, Szabelski P (2017) Hierarchical ordering in adsorbed overlayers of chiral tripod molecules with directional interactions. J Phys Chem C 121:410-420

38. Szabelski P, Nieckarz D, Rżysko W (2017) Influence of molecular shape and interaction anisotropy on the self-assembly of tripod building blocks on solid surfaces. Colloids Surfaces A 532:522-529

39. Szabelski P, Nieckarz D, Rżysko W (2017) Structure formation in 2D assemblies comprising functional tripod molecules with reduced symmetry. J Phys Chem C 121:25104-25117

40. Frenkel D, Smit B (1996) Understanding molecular simulation. Academic Press, London 